\title{
Harnessing preclinical mouse models to inform human clinical cancer trials
}

\author{
David H. Gutmann, ${ }^{1}$ Kim Hunter-Schaedle, ${ }^{2}$ and Kevin M. Shannon ${ }^{3}$
}

1Department of Neurology, Washington University School of Medicine, St. Louis, Missouri, USA. ${ }^{2}$ Children's Tumor Foundation, New York, New York, USA. ${ }^{3}$ Department of Pediatrics, UCSF, San Francisco, California, USA.

\begin{abstract}
The urgent need for better cancer treatments has stimulated interest in employing small-animal models to evaluate potential drug therapies. Robust mouse models of many human cancers have been generated using sophisticated technologies for engineering germ-line mutations. As we enter into an age of targeted therapeutics, these strains provide novel platforms for validating new anticancer drugs, assessing therapeutic index, identifying surrogate markers of tumor progression, and defining epigenetic and environmental influences on tumorigenesis.
\end{abstract}

The availability of strains of genetically engineered mice (GEM) that develop a spectrum of cancers similar to those found in humans offers an unprecedented opportunity to efficiently evaluate the efficacy and therapeutic index of novel anticancer therapies in preclinical models in advance of human trials. While straightforward in principle, executing preclinical studies in mice that allow for meaningful and immediate application to the treatment of human cancer is difficult. Moreover, the potential use of GEM cancer models to accelerate the process of bringing effective new treatments to patients is largely theoretical, as few examples exist in which mouse preclinical data has been successfully translated to clinical practice.

\section{The current development process for anticancer drugs}

Taking a drug from discovery to market is an arduous process that frequently takes longer than 15 years and costs more than $\$ 800$ million. Most agents that are advanced into early-phase human clinical trials fail. Recent advances in the fields of cancer biology and high-throughput screening have identified numerous potential molecular targets for drug discovery; however, most of the proteins and pathways deregulated in cancer cells also have essential roles in normal cells. It is therefore difficult to predict when a drug will prove tumor-selective. Moreover, developing new therapies against specific molecular abnormalities in well-defined subsets of cancers can be prohibitively expensive. The use of GEM cancer models as an initial "filter" to identify tumors and molecular targets that, when inhibited, will selectively kill tumor cells is one potential strategy for streamlining the overall process of cancer drug development.

\section{Preclinical mouse models of human cancer}

Numerous small-animal models of human cancer have been generated. These include inbred strains that spontaneously develop cancer (1-4), rodents in which cancer is caused by intrauterine or postnatal exposure to chemical mutagens (5-9), and mice in

Nonstandard abbreviations used: APL, acute promyelocytic leukemia; ATRA, all-trans-retinoic acid; Ftase, farnesyltransferase; FTI, farnesyltransferase inhibitor; GEM, genetically engineered mouse; MPNST, malignant peripheral nerve sheath tumor; MTD, maximally tolerated dose; NF1, neurofibromatosis 1 ; PK/PD, pharmacokinetic and pharmacodynamic; PML, promyelocytic leukemia; RARA, retinoic acid receptor alpha.

Conflict of interest: The authors have declared that no conflict of interest exists. Citation for this article: J. Clin. Invest. 116:847-852 (2006). doi:10.1172/JCI28271. which tumors are produced by viral or bacterial infection (10-13). In addition, xenograft models that were generated by directly implanting cancer cell lines established from human tumors into mice have been widely used for drug discovery (14-17). The major limitations of these explant models are the requirement for an immunocompromised host and the inability of these models to fully recapitulate the complex relationship between the tumor and its microenvironment (e.g., angiogenesis). Most importantly, the ability of xenografts to accurately predict drug efficacy in human cancer patients has been disappointing (18).

GEM cancer models are becoming increasingly sophisticated in their ability to accurately mimic the histology and biological behavior of human cancers. Numerous tissue-specific GEM models have been developed that exhibit many biologic hallmarks of human cancer, including angiogenesis and stromal interactions, as well as similar histopathologic and genetic abnormalities (19). The major advantages of GEM models are that: (a) the initiating genetic event is known; (b) the mice are immunocompetent; and (c) the tumors develop spontaneously in their appropriate tissue compartments. Moreover, GEM cancer models, which allow assessment of therapeutic efficacy on a uniform genetic background, are particularly useful for performing preclinical studies of rare cancers and for assessing synergy between therapeutic agents. They can also potentially provide the tools needed to learn more about the histologic and biochemical effects of specific agents prior to human testing.

While GEM models offer many advantages, the cancers typically arise from genetic events that are expressed simultaneously in many cells throughout an animal or in an entire tissue. By contrast, most human tumors are believed to arise from single cells or from a small population of mutant cells. To overcome this limitation, strategies have been developed that allow mutant alleles to be expressed in small populations of cells in vivo $(20,21)$.

\section{Opportunities to employ mouse models}

The availability of robust GEM models facilitates a detailed analysis of human cancer that cannot be easily accomplished by studying primary human tumors (see Opportunities provided by employing GEM cancer models). First, the ability to more effectively treat human cancers requires a detailed understanding of molecular and cellular pathogenesis to identify specific molecular targets. Second, there is also a great need to define those individuals at greatest risk for developing cancer as well as those most likely to respond to any 
given therapeutic regimen. These studies require large numbers of individuals and are often not possible for less common cancers. Last, the identification of surrogate markers of tumor formation and early response to therapy, which would have tremendous impact on current treatment strategies, is another unmet need.

Evaluation of standard human antitumor therapies. One of the often neglected uses of GEM cancer models is the validation of conventional therapies employed for the treatment of cognate tumors in humans. For example, accurate GEM models of astrocytoma or pancreatic cancer should ideally respond to the same treatments currently used to treat these cancers (i.e., temozolomide and gemcitibene, respectively). In addition, GEM models afford the opportunity to define the mechanism(s) underlying the antitumor effects. Tumors from mice treated with anticancer therapies can be analyzed to determine whether regression results from decreased cell growth, increased cell death, decreased tumor angiogenesis, or necrosis. Failure to observe any effects on GEM tumors may reflect problems with bioavailability (e.g., inability to cross the blood-brain barrier), differences in the metabolic processing of drugs in rodents (e.g., pharmacokinetic and pharmacodynamic $[\mathrm{PK} / \mathrm{PD}]$ issues), and/or genetic differences between mouse strains that dictate the response to therapy (e.g., modifier loci).

Experience with a mouse model of acute promyelocytic leukemia (APL) suggests that GEM models respond to human cancer treatments and can be used to improve therapy. In APL, blasts are arrested at the promyelocytic stage of differentiation due to chromosomal translocations that fuse the retinoic acid receptor alpha $(R A R A)$ gene to a variety of partner genes including promyelocytic leukemia (PML) and promyelocytic leukemia zinc finger (PLZF). Alltrans-retinoic acid (ATRA) induces complete remissions in approximately $80 \%$ of patients with APL who have a PML-RARA translocation by relieving the differentiation block (22) but does not induce remission in those individuals with PLZF-RARA fusions (23). Similarly, ATRA induces remissions in $P M L-R A R A$ transgenic mice but is ineffective in a PLZF-RARA strain that also develops APL (24). In addition, mouse models of APL have been harnessed to test new therapeutic approaches such as arsenic trioxide $\left(\mathrm{As}_{2} \mathrm{O}_{3}\right)$ and the potential synergy between ATRA and $\mathrm{As}_{2} \mathrm{O}_{3}(25,26)$.

The role of specific cancer genes. GEM strains have been generated that model the inactivation of genes mutated in inherited cancer syndromes (e.g., neurofibromatosis 1 [NF1], NF2, APC), in sporadic cancers (e.g., KRAS, PML-RARA), and in both types of cancer (e.g., TP53) (27-46). GEM models based on these tumor suppressors and oncogenes provide unique opportunities to clearly define the causative role of each of these genetic changes in tumor formation and progression. This information is critical for the design of targeted (biologically based) therapies for individual cancers with these specific tumor-associated mutations.
Target validation. GEM cancer models can be used to determine whether the success or failure of a given therapy reflects the ability of the drug to reach the tumor and inhibit its target. An illustrative example of how GEM cancer models can provide insights into mechanisms of drug activity comes from studies that evaluated the efficacy and putative biochemical targets of farnesyltransferase (FTase) inhibitors (FTIs). Ras processing is initiated by cytosolic prenyltransferases, which attach either a farnesyl or geranylgeranyl isoprenoid lipid to the thiol group of the cysteine. Geranylgeranyl transferase 1 (GGTase-1) and FTase catalyze the transfer of isoprenoid groups, which are donated by geranylgeranyl pyrophosphate and farnesyl pyrophosphate, respectively. FTIs were developed as cancer therapeutics based on their potential as Ras inhibitors in xenograft models. However, KRAS and NRAS are also good GGTase- 1 substrates and are processed by this enzyme when FTase is inhibited. Preclinical studies of the efficacy of FTIs gave variable results in transgenic mouse models of breast cancer induced by expressing oncogenic HRAS or KRAS from the murine mammary tumor virus promoter (47-49) and in a model of myeloproliferative disease induced by inactivating the Nf1 tumor suppressor (50), which encodes a GTPase-activating protein that negatively regulates RAS signaling. Importantly, careful biochemical investigation of tumor tissues from these mouse models unequivocally showed no inhibition of KRAS or NRAS processing at the maximally tolerated dose (MTD) of FTI. Based on these data, it was concluded that any therapeutic effects of FTIs were due to "off-target" activities that were not related to the original goal of inhibiting hyperactive RAS.

Defining the discrete steps of tumorigenesis. GEM cancer models can be used to dissect the cellular and molecular changes associated with each stage of neoplasia, including tumor formation, tumor maintenance, and malignant progression. Studies focused on defining the events associated with tumor formation in multistep cancers are essentially chemoprevention investigations. Direct chemoprevention studies in people at risk for cancer are difficult, owing to the genetic heterogeneity in human populations and the difficulties in accurately measuring exposure, which necessitate large and enormously expensive long-term studies. By contrast, experiments in GEM cancer models can be performed on a uniform genetic background in which environmental exposures are rigorously controlled. GEM cancer models have been employed to establish causal relationships with environmental exposures (e.g., asbestos in mesothelioma, tobacco and lung cancer; diet in colon cancer) (51-55).

The ability of a tumor to continue to survive and proliferate in an otherwise inhospitable environment requires additional molecular and cellular changes. Studies of tumor maintenance are typically focused on defining the key signals required for these processes and form the basis for targeted chemotherapy. Studies in GEM models

\section{Opportunities provided by employing GEM cancer models}

Provide initial "filter" to identify molecular targets that, when inhibited, kill cancer cells Investigate mechanisms underlying responsiveness and resistance to conventional cancer therapies Define discrete steps of tumorigenesis Determine the role of the microenvironment in tumor formation and progression Identify surrogate markers of tumor growth and response to therapy Define epigenetic and environmental influences on tumorigenesis 
and in human patients have implied that molecular changes important for cancer formation are also necessary for maintenance. For example, studies in which tetracycline-regulatable alleles of oncogenic RAS and MYC were "shut off" in established tumors resulted in dramatic tumor regression (56-58). Furthermore, the emergence of imatinib-resistant mutant alleles of $B C R-A B L$ in patients with chronic myeloid leukemia $(59,60)$ argues strongly that the cancerinitiating mutation remains central to the tumor's growth advantage. However, other data suggest that cancer cells can escape from dependence on the initiating oncogenic lesion under some circumstances $(61,62)$. The exact mechanisms underlying "tumor escape" have not been fully elucidated; but they may reflect a change in the histologic phenotype of the tumor, loss of expression of the initiating oncogene, or the acquisition of additional genetic changes (63). The ability of some cancers to free themselves from dependence on the initiating molecular event likely has implications for the design of targeted therapies for recurrent tumors.

Tumors frequently evolve from a benign neoplastic lesion to a more malignant cancer. This progression involves the acquisition of additional genetic changes, which also serve as targets for chemotherapeutic drug design. For example, during the progression to malignant cancer, some low-grade astrocytomas somatically acquire a constitutively active version of the EGFR. This signature genetic event formed the basis for the development of targeted therapies directed against this mutant EGFR in both mice and humans $(64,65)$. GEM models were important in demonstrating that the EGFR mutation is a causative genetic change that accelerates malignant transformation $(66,67)$.

Tumor microenvironment. GEM cancer models have been powerful tools for examining the contribution of the tumor microenvironment to tumor formation. Studies of peripheral and central nervous system tumors in a mouse model of the NF1 familial cancer syndrome demonstrated that tumor formation requires that loss of Nf1 expression in Schwann cells (neurofibromas) or astrocytes (optic glioma) occur in the context of a heterozygous germline $N f 1$ mutation $(43,44)$. These data demonstrate that heterozygous $N f 1$ mutant cells in the microenvironment of preneoplastic lesions participate in tumorigenesis. Nonmalignant stromal cells also contribute to mammary carcinoma, in which loss of TGF- $\beta$ receptor expression in fibroblasts promotes mammary ductal carcinoma growth and invasion by upregulating specific signaling networks $(68,69)$. Last, angiogenesis plays a fundamental role in tumor formation and progression and has formed the biological basis for numerous clinical trials using antiangiogenic therapies $(70,71)$. GEM models have been instructive in defining the molecular basis for new blood vessel formation by tumors and the impact of angiogenesis on tumor progression $(72,73)$.

Radiologic and serum biomarkers. The ability to define individuals at high risk of developing cancer and the ability to noninvasively monitor disease burden during and after cancer treatment have substantial implications for clinical practice. GEM models have been employed to identify serum biomarkers for cancer using advanced proteomics methods. While these studies are still in their early phases of discovery, one serum biomarker has been identified for murine prostate cancer that correlated well with tumor weight and response to hormone therapy (74). In addition to serum biomarkers, MRI has recently been evaluated for its ability to provide information regarding therapeutic efficacy in brain tumors. MRI of mice bearing brain tumors demonstrated that the tissue diffusion values obtained early after standard chemotherapy correlated with tumor response (75). These results prompted an investigation of human brain tumors, which showed that tissue diffusion values obtained 3 weeks after the initiation of chemotherapy could predict patient response (76). Similar to serum biomarkers, the ability of MRI to define patients with recurrent disease or who do not respond to first-line therapy would allow for early intervention and the administration of alternative therapies.

Modifier genes. Unlike humans, GEM models can be generated on homogeneous genetic backgrounds, which greatly facilitate identifying modifier genes that influence the incidence or clinical behavior of specific cancers. Numerous candidate genetic loci have been found that influence tumor number and size in mouse lung and colon cancer (77-79) as well as tumor type in mice harboring identical genetic mutations. For example, the tumor spectrum in mice harboring mutations in the p53 and Nf1 genes is dictated by the genetic background, which led to the identification of a locus on mouse chromosome 11 that determined susceptibility to astrocytoma (80). Last, genes that function to identify DNA polymerase errors during DNA replication (DNA mismatch repair genes) have been shown to modify colon cancer tumor burden and survival in GEM (81-83).

\section{Performing preclinical studies in mice}

Evaluating conventional cancer therapies in buman patients. There are well-established paradigms for testing new drugs in human patients. New agents are typically evaluated in 3 phases. As the primary goal of a phase 1 trial is to determine the MTD of a drug, these studies typically involve administering a single agent to patients with a variety of different tumor types who have failed to respond to standard therapies. Phase 2 trials are designed to measure response rates in a group of patients with refractory or recurrent cancers treated at the MTD. Responses are traditionally reported as "complete" (objective regression of all detectable lesions), "partial" (some regression), or "mixed" (regression of some lesions with growth of others). Compounds that show significant promise in phase 2 studies are advanced to randomized phase 3 trials, in which the new drug is compared, either alone or in combination with other agents, to the "standard" treatment for a specific cancer. In contrast to phase 1 and phase 2 trials, phase 3 studies include newly diagnosed patients and are frequently performed in the setting of cooperative multi-institutional networks. Because phase 2 and phase 3 studies are logistically challenging, expensive, and time consuming, pharmaceutical and biotechnology companies are understandably most interested in testing agents that might be approved to treat patients with common cancers. Importantly, phase 1 trials in patients with refractory cancers may not accurately mimic response rates in persons with de novo disease, and it has been difficult to test drug combinations in phase 1 and phase 2 trials. GEM cancer models offer the possibility of overcoming these 2 problems.

Using GEM cancer models to investigate responsiveness and resistance to conventional anticancer agents. Relatively few studies of conventional cytotoxic agents have been performed in GEM models. This is due, in part, to the fact that many investigators who generate GEM cancer models lack expertise in performing preclinical studies. Although much less expensive than human clinical trials, investigating drugs in mice is challenging due to factors that include the need to generate and maintain cohorts of mice that spontaneously develop tumors, difficulties in assessing the responses of tumors that can only be visualized by small-animal 


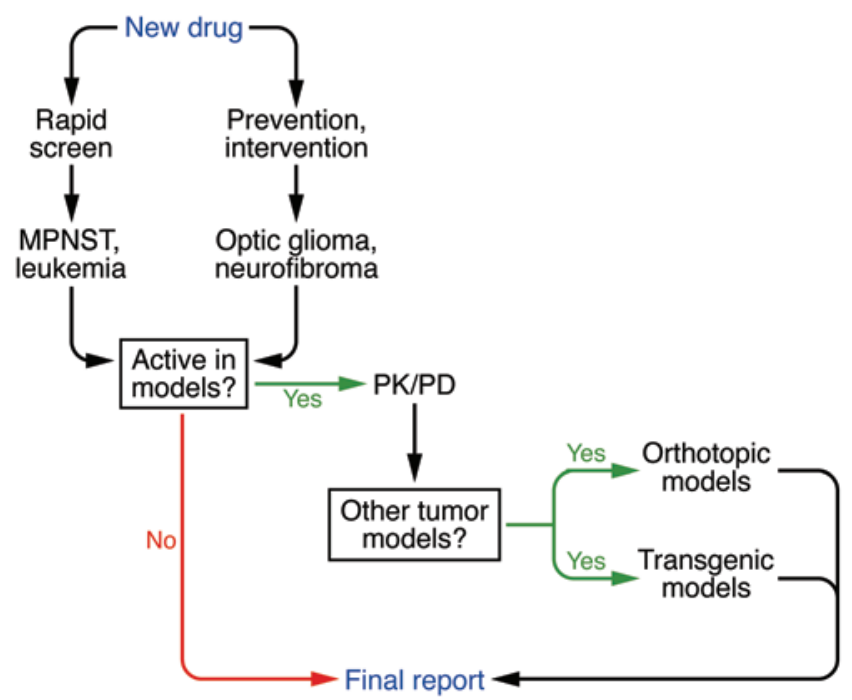

\section{Figure 1}

Use of GEM tumor models as "filters" to select agents for human clinical trials. One strategy has been proposed for use in the Nf1 GEM models community, which involves the evaluation of new therapies in multiple mouse strains. New drugs would be rapidly screened using MPNST and leukemia GEM models for efficacy (therapeutic index), target validation, and potential "off-target" effects. These GEM models would be utilized for initial evaluation, based on the rapid growth of the tumors and the relative ease of measuring tumor growth. Drugs that exhibit activity in these models would be further analyzed in detailed PK/PD studies in other tumor models, such as orthotopic tumor explant models and transgenic mice harboring specific deregulated cancerassociated molecules or pathways. Optic glioma and neurofibroma (plexiform neurofibroma) GEM models may be better suited for chemoprevention studies as well as investigations of drugs that target specific cells in the tumor microenvironment (e.g., microglia and mast cells). Collectively, the combined use of each of the available robust preclinical GEM models would afford researchers the opportunity to comprehensively evaluate drugs prior to considering human clinical trials. Adapted with permission from a summary presentation by Susan Blaney, Cold Spring Harbor Laboratories, Banbury Center conference on "Barriers and Solutions in the Use of Mouse Models to Develop Therapeutic Strategies for Neurofibromatosis-Associated Tumors," November 3-5, 2005.

imaging (e.g., MRI), and the limited availability of laboratory support to measure $\mathrm{PK} / \mathrm{PD}$ endpoints. Similarly, it is important to understand why some human tumors are inherently insensitive to chemotherapeutic agents, while other cancers initially respond but later become resistant when patients relapse. Pioneering studies in an $E \mu-M y c$ B cell lymphoma model have shown that some of the genetic lesions that contribute to cancer, such as Tp53 inactivation or deregulated $B c l 2$ expression, also modulate resistance to chemotherapeutic agents $(84,85)$.

Evaluating molecularly targeted inhibitors in humans and in GEM models. Some traditional strategies for evaluating new cancer therapeutics are being reconsidered as more specific agents are developed (86). Target inhibition, rather than overt clinical toxicity (e.g., MTD), may represent a better endpoint for phase 1 testing of drugs with a well-defined biochemical target. Additionally, there are now many examples that underscore the importance of preselecting patients with specific molecular abnormalities for targeted therapies trials. In this regard, the beneficial effects of ATRA are largely limited to APL; the efficacy of imatinib correlates with biochemical inhibition of specific mutant kinases (BCR-ABL, ckit, and PDGF); and the presence of activating EGFR mutations predicts clinical responsiveness to gefitinib in lung cancer $(25$, 87-91). In a recent study, coexpression of a mutant EGFR receptor and an intact PTEN gene correlated with the response of highgrade malignant astrocytoma to EGFR inhibitors (92). Similarly, although RAS and BRAF mutations both encode proteins that deregulate MEK/ERK signaling in melanoma, cancer cell lines with BRAF mutations are highly sensitive to MEK inhibitors, whereas cells with RAS mutations are not (93).

Harnessing GEM cancer models to enhance the development of new therapies. Academic researchers, pharmaceutical companies, government agencies, and patient advocacy groups have all expressed concern about the apparent "disconnect" between our growing understanding of cancer biology and the relatively few instances in which these advances have been successfully translated into better cancer treatments. The authors recently participated in a meeting that examined how mouse models of tumors that develop in persons with NF1 and NF2 could be efficiently employed to inform human clinical trials ("Barriers and Solutions in the Use of Mouse Models to Develop Therapeutic Strategies for Neurofibromatosis-Associated Tumors," Banbury Center, Cold Spring Harbor Laboratories, November 3-5, 2005). Individuals with NF1 are predisposed to the development of specific benign and malignant tumors, including cutaneous and plexiform neurofibromas, low-grade astrocytoma, juvenile myelomonocytic leukemia (JMML), and malignant peripheral nerve sheath tumor (MPNST), while persons with NF2 develop schwannoma, meningioma, and ependymoma (94). Because NF1- and NF2-associated tumors are relatively uncommon, pharmaceutical and biotechnology companies are not actively engaged in developing drugs for these specific indications. However, the molecular genetics of human NF1 and NF2 are understood in detail, and elegant mouse models of most NF-associated tumors are available. Many companies are developing drugs that interfere with components of the RAS signaling network, which might prove effective in some NF1-associated tumors. Unfortunately, performing clinical trials in NF1 patients is difficult for a variety of reasons, including the slow and predictable growth rates of many of these tumors, the propensity to affect children, and relatively small patient numbers. GEM models of JMML and MPNST are characterized by rapid growth and relative ease of measuring treatment responses $(45,46,95,96)$. These in vivo models, and tumor cells from these mice, could be used to rapidly screen candidate drugs for a beneficial therapeutic index, and promising agents might be investigated further by performing detailed PK/PD studies. By contrast, evaluating therapeutics in the existing neurofibroma and optic glioma GEM models is more difficult due to their relatively slow growth rates and requirement for small-animal imaging $(43,44)$. These models might be more useful for studies of preventive agents or for "front-line" preclinical studies of compounds that target cells in the tumor microenvironment. The overall goal of this type of strategy (Figure 1) is to optimally employ the available GEM tumor models as "filters" to select agents for human trials that have the greatest likelihood of succeeding in the clinic. In this proposed strategy, new drugs could be rapidly screened for efficacy, target validation, and potential "off-target" effects in GEM models that lend themselves well to rapid throughput (e.g., Nf1 MPNST and leukemia GEM models). Drugs active in these paradigms would be further studied to define 
their bioavailability and pharmacokinetics in other GEM model systems (e.g., orthotopic transplant or transgenic mouse models). Secondary evaluation of candidate drugs would then entail the use of additional GEM strains (e.g., Nf1 optic glioma and neurofibroma GEM models) in which tumor microenvironment plays an important role in cancer formation. These latter GEM models are uniquely suited for chemoprevention studies as well as for examining drugs directed against specific cell types in the tumor microenvironment (e.g., immune system cells, endothelial cells). The combined use of multiple complementary preclinical model systems provides an excellent opportunity to comprehensively evaluate lead compounds under conditions that closely approximate the human condition prior to the initiation of human clinical trials.

Given the pressing need to develop new cancer therapies, it is important to establish preclinical testing paradigms that provide the greatest opportunities to optimally translate results obtained in GEM cancer models into the clinic. We recommend that investigators take advantage of the multiple complementary GEM can- cer models now available to evaluate new agents in order to best inform subsequent human clinical trials.

\section{Acknowledgments}

We apologize to investigators whose work we were not able to discuss in this focused review. Our research in this area is supported by grants from the US Army, American Cancer Society, James S. McDonnell Foundation, and NIH. The impetus for writing this review was the Children's Tumor Foundation-sponsored meeting "Barriers and Solutions in the Use of Mouse Models to Develop Therapeutic Strategies for Neurofibromatosis-Associated Tumors," held at the Banbury Center, Cold Spring Harbor Laboratories, November 3-5, 2005.

Address correspondence to: David H. Gutmann, Department of Neurology, Washington University School of Medicine, Box 8111, 660 S. Euclid Avenue, St. Louis, Missouri 63110, USA. Phone: (314) 362-7379; Fax: (314) 362-2388; E-mail: gutmannd@neuro.wustl.edu.
1. Lubet, R.A., Zhang, Z., Wang, Y., and You, M. 2004 Chemoprevention of lung cancer in transgenic mice. Chest. 125:144S-147S.

2. Serano, R.D., Pegram, C.N., and Bigner, D.D. 1980 Tumorigenic cell culture lines from a spontaneous VM/Dk murine astrocytoma (SMA). Acta Neuro pathol. (Berl.). 51:53-64.

3. Pattengale, P.K., and Frith, C.H. 1983. Immunomorphologic classification of spontaneous lymphoid cell neoplasms occurring in female BALB/c mice. J. Natl. Cancer Inst. 70:169-179.

4. Huebner, R.J., et al. 1976. Prevention of spontaneous leukemia in AKR mice by type-specific immunosuppression of endogenous ecotropic virogenes. Proc. Natl. Acad. Sci. U. S. A. 73:4633-4635.

5. Zook, B.C., and Simmens, S.J. 2005. Neurogenic tumors in rats induced by ethylnitrosourea. Exp. Toxicol. Pathol. 57:7-14.

6. Di Bisceglie, A.M., Osmack, P., and Brunt, E.M 2005. Chemoprevention of hepatocellular carcinoma: use of tamoxifen in an animal model of hepatocarcinogenesis. J. Lab. Clin. Med. 145:134-138.

7. Thompson, H.J., and Singh, M. 2000. Rat models of premalignant breast disease. J. Mammary Gland Biol. Neoplasia. 5:409-420.

8. Nagao, M., Ushijima, T., Toyota, M., Inoue, R., and Sugimura, T. 1997. Genetic changes induced by heterocyclic amines. Mutat. Res. 376:161-167.

9. Sugiyama, T., Osaka, M., Koami, K., Maeda, S., and Ueda, N. 2002. 7,12-DMBA-induced rat leukemia: a review with insights into future research. Lenk. Res. 26:1053-1068.

10. Pritchard, D.M., and Przemeck, S.M. 2004. Review article: how useful are the rodent animal models of gastric adenocarcinoma? Aliment. Pharmacol. Ther. 19:841-859.

11. Meuwissen, R., and Berns, A. 2005. Mouse models for human lung cancer. Genes Dev. 19:643-664.

12. Jamerson, M.H., Johnson, M.D., and Dickson, R.B. 2004. Of mice and Myc: c-Myc and mammary tumorigenesis. J. Mammary Gland Biol. Neoplasia. 9:27-37.

13. Abate-Shen, C., and Shen, M.M. 2002. Mouse models of prostate carcinogenesis. Trends Genet. 18:S1-S5.

14. Giannini, C., et al. 2005. Patient tumor EGFR and PDGFRA gene amplifications retained in an invasive intracranial xenograft model of glioblastoma multiforme. Neuro-oncology. 7:164-176.

15. Pieper, R.O. 2003. Defined human cellular systems in the study of glioma development. Front. Biosci. 8:s19-s27.

16. Singh, S.K., et al. 2004. Identification of human brain tumour initiating cells. Nature. 432:396-401.
17. Frijhoff, A.F., Conti, C.J., and Senderowicz, A.M. 2004. Advances in molecular carcinogenesis: current and future use of mouse models to screen and validate molecularly targeted anticancer drugs. Mol. Carcinog. 39:183-194.

18. Oost, T.K., et al. 2004. Discovery of potent antagonists of the antiapoptotic protein XIAP for the treatment of cancer. J. Med. Chem. 47:4417-4426.

19. National Cancer Institute. MMHCC cancer models database. http://cancermodels.nci.nih.gov.

20. Jackson, E.L., et al. 2001. Analysis of lung tumor initiation and progression using conditional expression of oncogenic K-ras. Genes Dev. 15:3243-3248.

21. Fisher, G.H., et al. 1999. Development of a flexible and specific gene delivery system for production of murine tumor models. Oncogene. 18:5253-5260.

22. Tallman, M.S. 1998. Therapy of acute promyelocytic leukemia: all-trans retinoic acid and beyond. Leukemia. 12(Suppl. 1):S37-S40.

23. Licht, J.D., et al. 1995. Clinical and molecular characterization of a rare syndrome of acute promyelocytic leukemia associated with translocation (11;17). Blood. 85:1083-1094.

24. He, L.Z., et al. 1998. Distinct interactions of PMLRARalpha and PLZF-RARalpha with co-repressors determine differential responses to RA in APL. Nat. Genet. 18:126-135.

25. Rego, E.M., He, L.Z., Warrell, R.P., Jr., Wang, Z.G., and Pandolfi, P.P. 2000. Retinoic acid (RA) and As2O3 treatment in transgenic models of acute promyelocytic leukemia (APL) unravel the distinct nature of the leukemogenic process induced by the PML-RARalpha and PLZF-RARalpha oncoproteins. Proc. Natl. Acad. Sci. U. S. A. 97:10173-10178.

26. Look, A.T. 1998. Arsenic and apoptosis in the treatment of acute promyelocytic leukemia. J. Natl. Cancer Inst. 90:86-88.

27. Zhu, Y., et al. 2005. Inactivation of NF1 in CNS causes increased glial progenitor proliferation and optic glioma formation. Development. 132:5577-5588.

28. Shannon, K.M., Le Beau, M.M., Largaespada, D.A., and Killeen, N. 2001. Modeling myeloid leukemia tumor suppressor gene inactivation in the mouse. Semin. Cancer Biol. 11:191-200.

29. Reilly, K.M., Loisel, D.A., Bronson, R.T., McLaughlin, M.E., and Jacks, T. 2000. Nf1;Trp53 mutant mice develop glioblastoma with evidence of strainspecific effects. Nat. Genet. 26:109-113.

30. Kalamarides, M., et al. 2002. Nf2 gene inactivation in arachnoidal cells is rate-limiting for meningioma development in the mouse. Genes Dev. 16:1060-1065

31. Giovannini, M., et al. 2000. Conditional biallelic Nf2 mutation in the mouse promotes manifestations of human neurofibromatosis type 2. Genes Dev. 14:1617-1630

32. Moser, A.R., et al. 1993. ApcMin, a mutation in the murine Apc gene, predisposes to mammary carcinomas and focal alveolar hyperplasias. Proc. Natl. Acad. Sci. U. S. A. 90:8977-8981.

33. Luongo, C., Moser, A.R., Gledhill, S., and Dove, W.F. 1994. Loss of Apc+ in intestinal adenomas from Min mice. Cancer Res. 54:5947-5952.

34. Johnson, L., et al. 2001. Somatic activation of the $\mathrm{K}$-ras oncogene causes early onset lung cancer in mice. Nature. 410:1111-1116.

35. Chan, I.T., et al. 2004. Conditional expression of oncogenic K-ras from its endogenous promoter induces a myeloproliferative disease. J. Clin. Invest. 113:528-538. doi:10.1172/JCI200420476.

36. Tuveson, D.A., et al. 2004. Endogenous oncogenic K-ras(G12D) stimulates proliferation and widespread neoplastic and developmental defects. Cancer Cell. 5:375-387.

37. Le Beau, M.M., Bitts, S., Davis, E.M., and Kogan, S.C. 2002. Recurring chromosomal abnormalities in leukemia in PML-RARA transgenic mice parallel human acute promyelocytic leukemia. Blood. 99:2985-2991.

38. Zhu, J., et al. 2005. A sumoylation site in PML/ RARA is essential for leukemic transformation. Cancer Cell. 7:143-153.

39. Marino, S., Vooijs, M., van Der Gulden, H., Jonkers, J., and Berns, A. 2000. Induction of medulloblastomas in p53-null mutant mice by somatic inactivation of $\mathrm{Rb}$ in the external granular layer cells of the cerebellum. Genes Dev. 14:994-1004.

40. Wijnhoven, S.W., et al. 2005. Mice expressing a mammary gland-specific $\mathrm{R} 270 \mathrm{H}$ mutation in the p53 tumor suppressor gene mimic human breast cancer development. Cancer Res. 65:8166-8173.

41. Olive, K.P., et al. 2004. Mutant p53 gain of function in two mouse models of Li-Fraumeni syndrome. Cell. 119:847-860.

42. Zhu, Y., et al. 2005. Early inactivation of p53 tumor suppressor gene cooperating with NF1 loss induces malignant astrocytoma. Cancer Cell. 8:119-130.

43. Zhu, Y., Ghosh, P., Charnay, P., Burns, D.K., and Parada, L.F. 2002. Neurofibromas in NF1: Schwann cell origin and role of tumor environment. Science. 296:920-922.

44. Bajenaru, M.L., et al. 2003. Optic nerve glioma in mice requires astrocyte $\mathrm{Nf1}$ gene inactivation and Nf1 brain heterozygosity. Cancer Res. 63:8573-8577.

45. Cichowski, K., et al. 1999. Mouse models of tumor development in neurofibromatosis type 1. Science. 286:2172-2176.

46. Vogel, K.S., et al. 1999. Mouse tumor model for 
neurofibromatosis type 1. Science. 286:2176-2179. 47. Kohl, N.E., et al. 1995. Inhibition of farnesyltransferase induces regression of mammary and salivary carcinomas in ras transgenic mice. Nat. Med. 1:792-797.

48. Lobell, R.B., et al. 2001. Evaluation of farnesyl protein transferase and geranylgeranyl:protein transferase inhibitor combinations in preclinical models. Cancer Res. 61:8758-8768.

49. Omer, C.A., et al. 2000. Mouse mammary tumor virus-Ki-rasB transgenic mice develop mammary carcinomas that can be growth-inhibited by a farnesyl:protein transferase inhibitor. Cancer Res. 60:2680-2688

50. Mahgoub, N., et al. 1999. In vitro and in vivo effects of a farnesyltransferase inhibitor on Nf1-deficient hematopoietic cells. Blood. 94:2469-2476.

51. Hecht, S.S. 2005. Carcinogenicity studies of inhaled cigarette smoke in laboratory animals: old and new. Carcinogenesis. 26:1488-1492.

52. Shen, Q., and Brown, P.H. 2005. Transgenic mouse models for the prevention of breast cancer. Mutat Res. 576:93-110.

53. Kavanaugh, C., and Green, J.E. 2003. The use of genetically altered mice for breast cancer prevention studies. J. Nutr. 133:2404S-2409S.

54. Altomare, D.A., et al. 2005. A mouse model recapitulating molecular features of human mesothelioma. Cancer Res. 65:8090-8095.

55. Fleury-Feith, J., et al. 2003. Hemizygosity of Nf2 is associated with increased susceptibility to asbestos-induced peritoneal tumours. Oncogene. 22:3799-3805

56. Chin, L., et al. 1999. Essential role for oncogenic Ras in tumour maintenance. Nature. 400:468-472.

57. Felsher, D.W., and Bishop, J.M. 1999. Reversible tumorigenesis by MYC in hematopoietic lineages. Mol. Cell. 4:199-207.

58. Jain, M., et al. 2002. Sustained loss of a neoplastic phenotype by brief inactivation of MYC. Science. 297:102-104

59. Gorre, M.E., et al. 2001. Clinical resistance to STI571 cancer therapy caused by BCR-ABL gene mutation or amplification. Science. 293:876-880.

60. Shah, N.P., et al. 2002. Multiple BCR-ABL kinase domain mutations confer polyclonal resistance to the tyrosine kinase inhibitor imatinib (STI571) in chronic phase and blast crisis chronic myeloid leukemia. Cancer Cell. 2:117-125.

61. Moody, S.E., et al. 2002. Conditional activation of $\mathrm{Neu}$ in the mammary epithelium of transgenic mice results in reversible pulmonary metastasis. Cancer Cell. 2:451-461.

62. Gunther, E.J., et al. 2003. Impact of p53 loss on reversal and recurrence of conditional Wnt-induced tumorigenesis. Genes Dev. 17:488-501.

63. Giuriato, S., and Felsher, D.W. 2003. How cancers escape their oncogene habit. Cell Cycle. 2:329-332.
64. Krishnan, S., Rao, R.D., James, C.D., and Sarkaria, J.N. 2003. Combination of epidermal growth factor receptor targeted therapy with radiation therapy for malignant gliomas. Front. Biosci. 8:e1-e13.

65. Kuan, C.T., Wikstrand, C.J., and Bigner, D.D. 2000 EGFRvIII as a promising target for antibody-based brain tumor therapy. Brain Tumor Pathol. 17:71-78.

66. Holland, E.C., Hively, W.P., DePinho, R.A., and Varmus, H.E. 1998. A constitutively active epidermal growth factor receptor cooperates with disruption of G1 cell-cycle arrest pathways to induce gliomalike lesions in mice. Genes Dev. 12:3675-3685.

67. Ding, H., et al. 2003. Oligodendrogliomas result from the expression of an activated mutant epidermal growth factor receptor in a RAS transgenic mouse astrocytoma model. Cancer Res. 63:1106-1113.

68. Cheng, N., et al. 2005. Loss of TGF-beta type II receptor in fibroblasts promotes mammary carcinoma growth and invasion through upregulation of TGF-alpha-, MSP- and HGF-mediated signaling networks. Oncogene. 24:5053-5068.

69. Bhowmick, N.A., et al. 2004. TGF-beta signaling in fibroblasts modulates the oncogenic potential of adjacent epithelia. Science. 303:848-851.

70. Folkman, J. 2003. Fundamental concepts of the angiogenic process. Curr. Mol. Med. 3:643-651.

71. Ellis, L.M. 2004. Preclinical data targeting vascular endothelial growth factor in colorectal cancer. Clin. Colorectal Cancer. 4(Suppl. 2):S55-S61.

72. Korc, M. 2003. Pathways for aberrant angiogenesis in pancreatic cancer. Mol. Cancer. 2:8.

73. Hoffman, R. 2002. Green fluorescent protein imaging of tumour growth, metastasis, and angiogenesis in mouse models. Lancet Oncol. 3:546-556.

74. Huizen, I.V., et al. 2005. Establishment of a serum tumor marker for preclinical trials of mouse prostate cancer models. Clin. Cancer Res. 11:7911-7919.

75. Chenevert, T.L., et al. 2000. Diffusion magnetic resonance imaging: an early surrogate marker of therapeutic efficacy in brain tumors. J. Natl. Cancer Inst. 92:2029-2036.

76. Moffat, B.A., et al. 2005. Functional diffusion map: a noninvasive MRI biomarker for early stratification of clinical brain tumor response. Proc. Natl. Acad. Sci. U. S. A. 102:5524-5529.

77. Malkinson, A.M. 2001. Primary lung tumors in mice as an aid for understanding, preventing, and treating human adenocarcinoma of the lung. Lung Cancer. 32:265-279.

78. Mullerova, J., and Hozak, P. 2004. Use of recombinant congenic strains in mapping disease-modifying genes. News Physiol. Sci. 19:105-109.

79. Haines, J., et al. 2005. Genetic basis of variation in adenoma multiplicity in ApcMin/+ Mom1S mice. Proc. Natl. Acad. Sci. U. S. A. 102:2868-2873.

80. Reilly, K.M., et al. 2004. Susceptibility to astrocytoma in mice mutant for Nf1 and Trp53 is linked to chromosome 11 and subject to epigenetic effects. Proc. Natl. Acad. Sci. U. S. A. 101:13008-13013.

81. Kuraguchi, M., et al. 2000. Tumor-associated Apc mutations in Mlh1-/- Apc1638N mice reveal a mutational signature of Mlh1 deficiency. Oncogene. 19:5755-5763.

82. Edelmann, W., et al. 1999. Tumorigenesis in Mlh1 and Mlh1/Apc1638N mutant mice. Cancer Res. 59:1301-1307.

83. Edelmann, W., et al. 1999. Mammalian MutS homologue 5 is required for chromosome pairing in meiosis. Nat. Genet. 21:123-127.

84. Hemann, M.T., et al. 2005. Evasion of the p53 tumour surveillance network by tumour-derived MYC mutants. Nature. 436:807-811.

85. Johnstone, R.W., Ruefli, A.A., and Lowe, S.W. 2002. Apoptosis: a link between cancer genetics and chemotherapy. Cell. 108:153-164.

86. Druker, B.J. 2002. Perspectives on the development of a molecularly targeted agent. Cancer Cell. 1:31-36.

87. He, L.Z., Merghoub, T., and Pandolfi, P.P. 1999. In vivo analysis of the molecular pathogenesis of acute promyelocytic leukemia in the mouse and its therapeutic implications. Oncogene. 18:5278-5292.

88. Hughes, T., and Branford, S. 2006. Molecular monitoring of BCR-ABL as a guide to clinical management in chronic myeloid leukaemia. Blood Rev. 20:29-41.

89. Carter, T.A., et al. 2005. Inhibition of drug-resistant mutants of ABL, KIT, and EGF receptor kinases. Proc. Natl. Acad. Sci. U. S. A. 102:11011-11016.

90. Apperley, J.F., et al. 2002. Response to imatinib mesylate in patients with chronic myeloproliferative diseases with rearrangements of the plateletderived growth factor receptor beta. N. Engl. J. Med. 347:481-487.

91. Abrams, T.J., Lee, L.B., Murray, L.J., Pryer, N.K., and Cherrington, J.M. 2003. SU11248 inhibits KIT and platelet-derived growth factor receptor beta in preclinical models of human small cell lung cancer. Mol. Cancer Ther. 2:471-478.

92. Mellinghoff, I.K., et al. 2005. Molecular determinants of the response of glioblastomas to EGFR kinase inhibitors. N. Engl. J. Med. 353:2012-2024.

93. Solit, D.B., et al. 2006. BRAF mutation predicts sensitivity to MEK inhibition. Nature. 439:358-362.

94. Gutmann, D.H., et al. 1997. The diagnostic evaluation and multidisciplinary management of neurofibromatosis 1 and neurofibromatosis 2. JAMA. 278:51-57.

95. Bollag, G., et al. 1996. Loss of NF1 results in activation of the Ras signaling pathway and leads to aberrant growth in haematopoietic cells. Nat. Genet. 12:144-148.

96. Le, D.T., et al. 2004. Somatic inactivation of Nf1 in hematopoietic cells results in a progressive myeloproliferative disorder. Blood. 103:4243-4250 